\title{
RESIDUAL LINEARITY FOR CERTAIN NILPOTENT GROUPS
}

\author{
P. MENAL
}

\begin{abstract}
In this note we consider relations between residual finiteness and residual linearity for a nilpotent group $G$. We show, amongst other things, that if the center and the commutator subgroup of $G$ are finitely generated and $G$ is residually linear, then $G$ is residually finite. Indeed the property which we use on linear groups is that linear groups satisfy the minimal condition on centralizers.
\end{abstract}

Let $\mathscr{\rho}$ be a group property. We recall that a given group $G$ is called residually $\mathcal{P}$ if it is a subdirect product of groups having the property $\mathcal{P}$. For any group $G$ let $R(G)$ be the intersection of all its normal subgroups of finite index. Thus $R(G)=\langle 1\rangle$ if and only if $G$ is residually finite. For any integer $n$ let $G^{n}$ be the subgroup of $G$ generated by the $n$th powers of elements of $G$. For abelian groups it is well known that $R(G)=\cap_{n>1} G^{n}$; if in addition the $p$-torsion of $G$ is bounded for each prime $p$, then $R(G)$ is a radicable group. We say that $G$ is residually linear if for each $1 \neq x \in G$ there exists a field $K$ and a homomorphism $\phi: G \rightarrow \operatorname{GL}(n, K)$ such that $\phi(x) \neq 1$. An abelian group is $\Re$ if it is a subdirect product of cyclic groups $C_{i}$ such that $C_{i} \cong \mathbf{Z}$ or $\left|C_{i}\right| \leqslant n$ for a fixed integer $n$. We will use the symbols $\Gamma_{n}(G)$ and $Z_{n}(G)$ for the terms in the lower and upper central series of $G$. If $X$ is a subset of the group $G$ we denote by $C_{G}(X)$ its centralizer.

The main result of this paper is

THEOREM I. (i) Let $G$ be a nilpotent residually linear group. If $\Gamma_{2}(G)$ is finitely generated and $Z_{1}(G)$ is $\Re$, then $G$ is residually finite.

(ii) There exists a nilpotent group of class 2 with $\Gamma_{2}(G)$ finitely generated and $Z_{1}(G)$ residually finite, such that it is residually linear but it is not residually finite.

(iii) There exists a nilpotent group of class 3 with $Z_{1}(G)$ cyclic, which is residually linear but it is not residually finite.

COROLlaRY. Let $G$ be a nilpotent group of class 2 with $Z_{1}(G)$ finitely generated. Then residually linear implies residually finite.

LEMMA I. Let $G$ be a nilpotent linear group. If $H$ is a normal subgroup of $G$ such that $H \cap Z_{1}(G)$ is finitely generated, then $H$ is finitely generated.

Received by the editors May 5, 1977.

AMS (MOS) subject classifications (1970). Primary 20D15, 20E25; Secondary $20 \mathrm{H} 20$.

Key words and phrases. Nilpotent group, residually finite, residually linear. 
Proof. We proceed by induction on the class $c$ of $G$, the case $c=1$ being obvious. Suppose that the elements $x_{1}, x_{2}, \ldots, x_{n}$ of $G$ span $G$ linearly, clearly

$$
Z_{1}(G)=C_{G}\left(x_{1}, x_{2}, \ldots, x_{n}\right)
$$

Let $[x, y]=x^{-1} y^{-1} x y$ denote commutators in $G$, then the map

$$
H \cap Z_{2}(G) \rightarrow\left(H \cap Z_{1}(G)\right) \times \cdots \times\left(H \cap Z_{1}(G)\right)
$$

in which $x \mapsto\left(\left[x, x_{1}\right], \ldots,\left[x, x_{n}\right]\right)$ is a group homomorphism with kernel $H \cap Z_{1}(G)$. Thus $\left(H \cap Z_{2}(G)\right) /\left(H \cap Z_{1}(G)\right)$ is finitely generated. Since $G$ is a linear group, $G / Z_{1}(G)$ is linear [5, Theorem 6.2] and we have that

$$
\left(H Z_{1}(G) / Z_{1}(G)\right) \cap Z_{1}\left(G / Z_{1}(G)\right) \cong\left(H \cap Z_{2}(G)\right) /\left(H \cap Z_{1}(G)\right)
$$

is finitely generated. By induction it follows that

$$
H Z_{1}(G) / Z_{1}(G) \cong H /\left(H \cap Z_{1}(G)\right)
$$

is finitely generated and the result is clear.

LEMMA 2. Let $G$ be a nilpotent linear group. Then the following are equivalent.

(i) If $x \in G$, the normal closure of $\langle x\rangle$ in $G$ is finitely generated.

(ii) $\Gamma_{2}(G)$ is finitely generated.

(iii) $G / Z_{1}(G)$ is finitely generated.

Proof. For arbitrary nilpotent groups (iii) implies (ii) [3, Corollary 3.19]. Trivially (ii) implies (i). Let $x_{1}, x_{2}, \ldots, x_{n}$ be elements of $G$ spanning $G$ linearly. If we suppose that the normal closure $F$ in $G$ of $\left\langle x_{1}, x_{2}, \ldots, x_{n}\right\rangle$ is finitely generated, the homomorphism

$$
Z_{2}(G) \rightarrow F \times \cdots \times F
$$

in which $x \mapsto\left(\left[x, x_{1}\right], \ldots,\left[x, x_{n}\right]\right)$ proves that $Z_{1}\left(G / Z_{1}(G)\right)$ is finitely generated. It follows from Lemma 1 that $G / Z_{1}(G)$ is finitely generated. This proves that (i) implies (iii).

LEMMA 3. Let $G$ be a nilpotent group such that $G / Z_{1}(G)$ is finitely generated. Then $G$ is residually finite if and only if $Z_{1}(G)$ is residually finite.

Proof. It suffices to show that $R\left(Z_{1}(G)\right)=R(G)$. Trivially $R\left(Z_{1}(G)\right) \subseteq$ $R(G)$. For if $N$ is a subgroup of $Z_{1}(G)$ of finite index, $N \triangleleft G$, so $G / N$ is finitely generated, nilpotent and hence residually finite [2, Theorem 2.1]. Thus $R(G) \subseteq N$ and $R(G) \subseteq R\left(Z_{1}(G)\right)$.

We remark that Lemma 3 is a trivial consequence of [4, Proposition 1]. However, the above is quite sufficient for our purposes.

Proposition 4. Let $G$ be a residually linear nilpotent group satisfying the following conditions.

(i) If $x \in G$, the normal closure of $\langle x\rangle$ in $G$ is finitely generated.

(ii) $G / \Gamma_{2}(G)$ is residually finite and for each prime $p$ its $p$-torsion is bounded.

Then $G$ is residually finite. 
Proof. Let $1 \neq x \in G$. We will prove that $x \notin R(G)$. Since $G$ is residually linear we can consider a homomorphism $\phi$ of $G$ into a linear group such that $\phi(x) \neq 1$. Let $\bar{G}=G /\left(\operatorname{Ker} \phi \cap \Gamma_{2}(G)\right)$. Since homomorphic images of $G$ satisfy (i) it follows from Lemma 2 that $\Gamma_{2}(G / \operatorname{Ker} \phi)$ and $(G / \operatorname{Ker} \phi) / Z_{1}(G / \operatorname{Ker} \phi)$ are finitely generated. Clearly $\bar{G} \hookrightarrow(G / \operatorname{Ker} \phi) \times$ $\left(G / \Gamma_{2}(G)\right)$. Then we see easily that $\Gamma_{2}(\bar{G})$ and $\bar{G} / Z_{1}(\bar{G})$ are finitely generated. Furthermore we have

$$
\bar{G} / \Gamma_{2}(\bar{G}) \cong G / \Gamma_{2}(G) \text {. }
$$

Thus by (ii) we conclude that the $p$-torsion of $\bar{G}$ is bounded for each prime $p$. Therefore $R\left(Z_{1}(\bar{G})\right)$ is a radicable group. But $\bar{G} / \Gamma_{2}(\bar{G})$ is residually finite so $R\left(Z_{1}(\bar{G})\right) \subseteq \Gamma_{2}(\bar{G})$. Since $\Gamma_{2}(\bar{G})$ is finitely generated, necessarily $R\left(Z_{1}(\bar{G})\right)$ $=\langle 1\rangle$. Now Lemma 3 implies that $\bar{G}$ is residually finite. Since $x \notin \operatorname{Ker} \phi \cap$ $\Gamma_{2}(G)$ we conclude that $x \notin R(G)$.

LEMMA 5. Let $G$ be $a \Re$ group and let $H$ be a finitely generated subgroup. Then $G / H$ is a $R$ group.

Proof. $G$ is a $R$ group hence $G \subseteq \Pi Z \times C$. Where $C$ is a bounded group and so a direct sum of cyclic groups [1, Theorem 17.2]. Since subgroups of $\Re$ groups are $R$ groups, we can assume $G=\Pi Z \times C$ in order to prove the lemma. Let $H$ be a finitely generated subgroup of $G$. Then there exist finitely generated subgroups $M \subseteq \Pi Z$ and $N \subseteq C$ such that $H \subseteq M \times N$. Every finitely generated subgroup of $\Pi Z$ can be embedded in a finitely generated direct summand of $\Pi Z$ [1, Theorem 19.2]. Clearly the same property holds for $C$. Therefore we may, in addition, suppose that $M \times M^{\prime}=\Pi Z$ and $N \times N^{\prime}$ $=C$ for some $M^{\prime} \subseteq \Pi Z$ and $N^{\prime} \subseteq C$. Now we have

$$
G / H \cong(M \times N / H) \times M^{\prime} \times N^{\prime}
$$

and the result follows.

We can now give the

Proof of THEOREM I. (i) By the proposition, we have only to prove that $G / \Gamma_{2}(G)$ is residually finite and the elements of $G / \Gamma_{2}(G)$ of finite order are of bounded order. We use induction on the class $c$ of $G$. If $c=1$ the result is trivial. Suppose $c>1$ and let $\bar{G}=G / Z_{1}(G)$. Trivially $\Gamma_{2}(\bar{G})$ is finitely generated. $Z_{1}(\bar{G})$ is $\Re$, since it is contained in a product $\Pi Z_{1}(G)$. By induction we have that $\bar{G} / \Gamma_{2}(\bar{G})$ is residually finite and its torsion is bounded. Since $Z_{1}(G)$ is $\mathcal{R}$ and $\Gamma_{2}(G)$ is finitely generated it follows from Lemma 5 that $Z_{1}(G) \Gamma_{2}(G) / \Gamma_{2}(G)$ is $\mathcal{R}$. Therefore we have that $G / \Gamma_{2}(G)$ is of torsion bounded. Thus $R\left(G / \Gamma_{2}(G)\right)$ is a radicable group contained in $Z_{1}(G) \Gamma_{2}(G) / \Gamma_{2}(G)$. Since $\mathcal{R}$ groups contain no nontrivial radicable groups we conclude that $G / \Gamma_{2}(G)$ is residually finite.

(ii) Let $p$ be a prime. Put

$$
\begin{array}{r}
G=\left\langle z_{i}, x_{i}, y_{i}, i=0,1, \ldots: z_{i+1}^{p}=z_{i},\right. \\
{\left[x_{i}, x_{j}\right]=\left[y_{i}, y_{j}\right]=\left[z_{i}, z_{j}\right]=\left[z_{i}, x_{j}\right]=\left[z_{i}, y_{j}\right]=1,} \\
\left.\left[x_{i}, y_{j}\right]=1 \text { if } i \neq j,\left[x_{i}, y_{i}\right]=z_{0}^{p^{i}}\right\rangle .
\end{array}
$$


$G$ is a nilpotent group of class 2 with $\Gamma_{2}(G)=\left\langle z_{0}\right\rangle$ and

$$
Z_{1}(G)=\left\langle z_{i}, i=0,1, \ldots\right\rangle \cong Q_{p}
$$

(where $Q_{p}$ is the group of all rational numbers whose denominators are powers of $p$ ). $Z_{1}(G)$ is residually finite however it does not satisfy $R$. We will prove that $G$ is not residually finite but it is residually linear. Suppose that $x \mapsto \bar{x}$ is a homomorphism of $G$ into a finite group $\bar{G}$. Then, by the finiteness of $\bar{G}$, there exist distinct integers $n, m$ with $\bar{x}_{n}=\bar{x}_{m}$. Thus $\overline{1}=\left[\bar{x}_{m}, \bar{y}_{m}\right]=$ $\left[\bar{x}_{n}, \bar{y}_{m}\right]=\bar{z} p^{m}$. Since a finite homomorphic image of $Q_{p}$ has no elements of order $p$, we have that $\bar{z}_{0}=\overline{1}$ so $z_{0} \in R(G)$. In fact $R(G)=Z_{1}(G)$. Let $K$ be a field containing the $p^{n}$-roots of the unity for any integer $n \geqslant 1$. In order to prove that $G$ is residually linear it suffices to show that the group $G_{n}=$ $G /\left\langle z_{0}^{p^{n}}\right\rangle$ is residually $K$-linear for any integer $n \geqslant 1$, since $\bigcap_{n>1}\left\langle z p^{n}\right\rangle=\langle 1\rangle$. It follows from the relations of $G$ that $Z_{1}\left(G_{n}\right)$ is $\mathbf{Z}\left(p^{\infty}\right)$ by a residually finite group. Therefore $Z_{1}\left(G_{n}\right)$ is residually $K$-linear. Clearly $Z_{1}\left(G_{n}\right)$ has finite index in $G_{n}$ so $G_{n}$ is residually $K$-linear and the result follows.

(iii) Let $p$ be a prime. Let $G$ be a group generated by $z, t_{i}, x_{i}, y_{i}, i=$ $1,2, \ldots$, subject to the relations

$$
\begin{aligned}
& {\left[x_{i}, x_{j}\right]=\left[y_{i}, y_{j}\right]=\left[t_{i}, t_{j}\right]=\left[z, x_{i}\right]=\left[z, t_{i}\right]=\left[z, y_{i}\right]=1,} \\
& {\left[x_{i}, y_{i}\right]=t_{i}^{p^{i}} z, \quad\left[x_{i}, y_{j}\right]=1 \quad \text { if } i \neq j,} \\
& {\left[t_{i}, x_{i}\right]=\left[t_{i}, y_{i}\right]=z^{p^{\prime}}, \quad\left[t_{i}, x_{j}\right]=\left[t_{i}, y_{j}\right]=1 \quad \text { if } i \neq j .}
\end{aligned}
$$

$G$ is a torsion free nilpotent group of class 3 with center $\langle z\rangle$. Let $x \mapsto \bar{x}$ be a homomorphism of $G$ into a finite group $\bar{G}$. Then, by finiteness of $\bar{G}$, there exist distinct integers $n, m$ with $\bar{y}_{n}=\bar{y}_{m}$. Thus

$$
\overline{1}=\left[\bar{t}_{m}, \bar{y}_{n}\right]=\left[\bar{t}_{m}, \bar{y}_{m}\right]=\bar{z}^{p^{m}} \text {. }
$$

Suppose that $\bar{z} \neq \overline{1}$. Then $h_{p}(\bar{z})$, the $p$-height of $\bar{z}$ in $\bar{G}$, is finite. Again there exist distinct integers $r, s>h_{p}(\bar{z})$, with $\overline{1}=\left[\bar{x}_{r}, \bar{y}_{s}\right]=\left[\bar{x}_{s}, \bar{y}_{s}\right]=\bar{t}_{s}^{p} \bar{z}$. Hence $\bar{z}=\left(\bar{t}_{s}^{-1}\right)^{p^{s}}$ and $s \leqslant h_{p}(\bar{z})$, a contradiction. Therefore we have shown that $z$ belongs to the kernels of all homomorphism of $G$ into finite groups so $z \in R(G)$. Finally we show that $G$ is residually $K$-linear, if $K$ contains, for every $n$, the $p^{n}$-roots of the unity. Define for each integer $n \geqslant 1$

$$
H_{n}=\left\langle t_{1}^{p^{n}}, t_{2}^{p^{n}}, \ldots, t_{n-1}^{p^{n}}, z^{p^{n}}, t_{m}^{p^{m}} z \text { for } m \geqslant n\right\rangle \text {. }
$$

Clearly $H_{n}$ is a normal subgroup of $G$ and $H_{n} \cap\langle z\rangle=\left\langle z^{p^{n}}\right\rangle$. Therefore $\cap_{n \geqslant 1} H_{n}=\langle 1\rangle$. Then it suffices to prove that the group $\bar{G}=G / H_{n}$ is residually $K$-linear. It is clear that

$$
\bar{G}=\left\langle Z_{1}(\bar{G}), \bar{x}_{1}, \ldots, \bar{x}_{n-1}, \bar{y}_{1}, \ldots, \bar{y}_{n-1}, \bar{t}_{1}, \ldots, \bar{t}_{n-1}\right\rangle .
$$

Furthermore, for $i=1,2, \ldots, n-1$, we have

$$
\begin{aligned}
\bar{t}_{i}^{p^{n}} & =\overline{1} \quad \text { so }\left[\bar{x}_{i}^{p^{n}}, \bar{t}_{i}\right]=\left[\bar{y}_{i}^{p^{n}}, \bar{t}_{i}\right]=\overline{1}, \\
{\left[\bar{x}_{i}^{p^{n}}, \bar{y}_{i}\right] } & =\left[\bar{x}_{i}, \bar{y}_{i}\right]^{p^{n}}\left[\bar{x}_{i}, \bar{y}_{i}, \bar{x}_{i}\right]^{p^{n}\left(p^{n}-1\right) / 2} \\
& =\bar{t}_{i}^{p^{i+n}} \bar{z}^{p^{n}} \bar{z}^{p^{2 i+n}\left(p^{n}-1\right) / 2}=\overline{1},
\end{aligned}
$$


similarly $\left[\bar{y}_{i}^{p^{n}}, \bar{x}_{i}\right]=\overline{1}$. These relations yield that $\bar{G} / Z_{1}(\bar{G})$ is a torsion group which is finite, since it is finitely generated. The result follows, since $Z_{1}(\bar{G})$ is residually $K$-linear.

I wish to thank Professor D. S. Passman who showed me an example which gave me the inspiration for this paper. Also I should like to thank the referee for his comments.

\section{REFERENCES}

1. L. Fuchs, Infinite abelian groups, Academic Press, New York, 1970.

2. K. W. Gruenberg, Residual properties of infinite soluble groups, Proc. London Math. Soc. (3) 7 (1957), 29-62.

3. R. B. Warfield, Jr., Nilpotent groups, Lecture Notes in Math., vol. 513, Springer-Verlag, Berlin, 1976.

4. B. A. F. Wehrfritz, A note on residual properties of nilpotent groups, J. London Math. Soc. (2) 5 (1972), 1-7.

5. , Infinite linear groups, Springer-Verlag, Berlin, 1973.

Secció de Matemàtiques, Universitat Autònoma de Barcelona, Barcelona, España 